\title{
Associative memories based on message-bearing optical modes in phase-conjugate resonators
}

\author{
Amnon Yariv and Sze-Keung Kwong \\ California Institute of Technology, Pasadena, California 91125
}

Received September 30, 1985; accepted January 6, 1986

\begin{abstract}
We describe in generic terms a class of associative memory that is based on (1) an oscillating message-bearing optical mode in a resonator containing a multimessage hologram, (2) image-selective (discriminatory) amplification of the desired picture in a photorefractive crystal, and (3) phase-conjugate reflection.
\end{abstract}

The recent few years have witnessed a strong interest in the area of associative memories. These are memories in which a stored piece of information can be retrieved whole when the probing input is only partially complete. The memory somehow finds that stored message, among many, which most closely resembles the input and puts it out.

There has been an increasing sense of awareness among workers in the field of phase-conjugate optics that this area may contain the clue for a holographic associative memory. This Letter is written with the purpose of describing some fundamental features of two-beam coupling and phase conjugation in photorefractive crystals $^{2-4}$ that are central to our initial probes in this direction. The hope is that these may stimulate other investigators to add and apply their specialties to the solution of this exciting problem.

The first basic concept is that of stored information as an electromagnetic mode. The idea was demonstrated in $1982^{5}$ in an experiment that showed that a laser oscillator with a severe distortion inside the resonator can oscillate nearly optimally provided that one of its mirrors is a phase-conjugate mirror. The situation is depicted in Fig. 1, which is reproduced from Ref. 6 . The distorted beam is time reversed by the phase-conjugate mirror (PCM) so that the beam to the left of the distortion is "clean," while that to the right is distorted. Now since one person's distortion is another person's information, we can associate the distorting element with a stored piece of information ("Information") and realize that the oscillation electromagnetic field to the right of the distortion is spatially modulated by the information.

For a useful memory device our intracavity storage element must store a large number of messages. This can be done if the element is a volume transmission hologram with a large number of (pre)recorded holograms, each one capable of giving rise to a unique oscillating mode. The next problem is how to use a partial (or a close) input to stimulate the oscillation of just the desired mode. Since in the competition between resonator modes for oscillation, the mode with the highest net gain dominates (to the near total exclusion of less favored modes), the question is how to provide preferential gain to the desired mode. One strong possibility is to use the spatial dependence of the power exchange in two-beam coupling. It was shown recently that the power transferred from a beam with a spatial field profile $E_{i}{ }^{\prime}(\mathbf{r})$ to a beam $E_{i}(\mathbf{r})$ is proportional to the integral ${ }^{7}$

$$
G \propto \int_{V_{\text {int }}} \frac{\left|E_{i}^{\prime}(\mathbf{r})\right|^{2}\left|E_{i}(\mathbf{r})\right|^{2}}{\mid \mathbf{r})\left.\right|^{2}+\left|E_{i}(\mathbf{r})\right|^{2}} \mathrm{~d}^{3} \mathbf{r} .
$$

This integral is maximum when

$$
E_{i}(\mathbf{r}) \propto E_{i}^{\prime}(\mathbf{r}) e^{i \phi(\mathbf{r})},
$$

where $\phi(\mathbf{r})$ is arbitrary. Condition (2) ensures that the zeros of both beams coincide, thus minimizing the "damage" to the integral. In practice, we may realize condition (2) in a contradirectional propagation of beams $E_{i}{ }^{\prime}(\mathbf{r})$ and $E_{i}(\mathbf{r})$. The spatial modulation of $E_{i}{ }^{\prime}(\mathbf{r})$ is due to some partial information. Beam $E_{i}(\mathbf{r})$ is one of the many potential stored information beams in the hologram. Beam $E_{i}(\mathbf{r})$ will emerge as that for which the overlap integral is maximum.

We have combined conceptually a number of key elements. To recall, these are

(1) An electromagnetic mode as a stored message;

(2) Spatial information dependence of the gain in photorefractive two-beam coupling;

(3) The highly nonlinear mode competition in laser oscillators, which results in the emergence of the desired mode provided that the partial (or imperfect) input provides preferential gain.

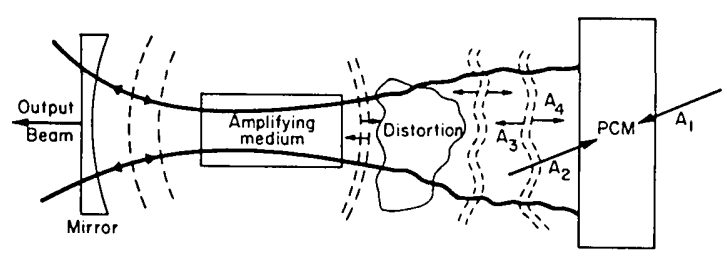

Fig. 1. A phase-conjugate reflector compensates for distortion caused by an image field, thus permitting a messagebearing oscillation inside an optical resonator. 
(a)

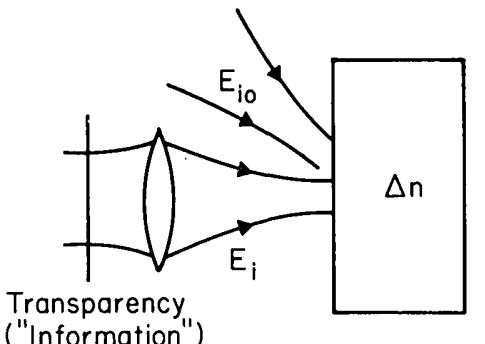

(b)

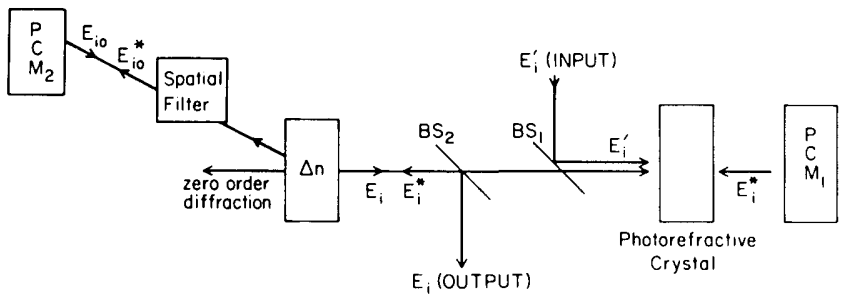

Fig. 2. (a) Information recorded on a fixed hologram $\Delta n$. $E_{i}$ is the beam carrying the information, and $E_{i o}$ is the reference beam. The angle between $E_{i o}(\mathbf{r})$ and $E_{i}(\mathbf{r})$ is sufficiently large to prevent spillover of information in the memory application into the zero-order mode. (See text discussion in connection with Fig. 2.) (b) A scheme for an optical associative memory: PCM's, the phase-conjugate mirrors; BS's, beam splitters; $\Delta n$, the prerecorded hologram; $E_{i}{ }^{\prime}(\mathbf{r})$, the pumping beam carrying partial information; $E_{i}(\mathbf{r})$, one of the oscillation modes of the resonator, which is excited preferentially by $E_{i}{ }^{\prime}(\mathbf{r})$.

We might view elements (1)-(3) as building blocks for associative memories.

One possible realization is shown in Fig. 2. Figure 2(a) shows how a message, $E_{i}$, is recorded holographically using reference $E_{i o}$. The direction of the reference beam is different for each stored message. The result is a volume index variation (hologram),

$$
\Delta n \propto \sum_{j=1}^{N}\left(E_{j o}{ }^{*} E_{j}+\text { c.c. }\right),
$$

where $N$ is the number of recorded messages. In Fig. 2(b) a resonator is shown that contains the hologram $\Delta n$ as an internal transmission element. The basic principle of operation is as follows. The oscillator is capable of oscillating in any one of the stored message modes $E_{j}$. Consider, for example, mode $i$. The laser beam $E_{i o}$ propagating from left to right diffracts off the stored hologram $\Delta n$ to yield a field term $E_{i o}(\mathbf{r}) E_{i o}{ }^{*}(\mathbf{r}) E_{i}(\mathbf{r})$. Since $E_{i o}(\mathbf{r})$ is essentially planar, the term $E_{i o}(\mathbf{r}) E_{i o} *(\mathbf{r})$ is a constant and the field that diffracted off from $\Delta n$ is proportional to $E_{i}(\mathbf{r})$. The wave $E_{i}(\mathbf{r})$, which passes through the crystal, is then reflected by the phase-conjugate mirror $\mathrm{PCM}_{1}$ to become $E_{i}^{*}(\mathbf{r})$. The wave $E_{i}^{*}(\mathbf{r})$ is traveling in the $-z$ direction. A pumping wave $E_{i}{ }^{\prime}(\mathbf{r})$, which enters the system through the reflection at the beam splitter $\mathrm{BS}_{1}$ and is propagating in the $z$ direction, amplifies $E_{i}^{*}(\mathbf{r})$ according to condition (1). The orientation of the crystal is chosen such that $(-z)$ is the amplification direction. The amplified $E_{i}^{*}(\mathbf{r})$ wave diffracts off the stored hologram $\Delta n$ to yield a field consisting of $N$ beams $\sum_{j} E_{j o}{ }^{*}(\mathbf{r}) E_{j}(\mathbf{r}) E_{i}^{*}(\mathbf{r})$. The diffracted beams are then passed through a low-pass spatial filter to obtain a field proportional to $\sum_{j} E_{j o}{ }^{*}(\mathbf{r})$. If $\mathrm{PCM}_{2}$ has thresholding characteristics so that it reflects only the strongest beam $E_{i o}{ }^{*}(\mathbf{r})$ that passed through the spatial filter, then the beam reflected from $\mathrm{PCM}_{2}$ is proportional to $\sum_{j} E_{j o}{ }^{*}(\mathbf{r})$, and we are back, self-consistently, to our starting condition. The output of the system is provided by beam splitter $\mathrm{BS}_{2}$ and is proportional to the stored message field $E_{i}(\mathbf{r})$.

Let us discuss the experimental feasibility of such a scheme. There are several methods that can be used to make multiple holograms. For example, 500 fixed holograms, each with more than $2.5 \%$ diffraction efficiency, in heated $\mathrm{Fe}$-doped $\mathrm{LiNbO}_{3},{ }^{8}$ and more than 10 holograms, each with $20 \%$ diffraction efficiency, in dichromated gelatin ${ }^{9}$ were reported separately. On the other hand, a phase-conjugate mirror with a gain of 100 was reported in photorefractive $\mathrm{BaTiO}_{3}$ crystals. ${ }^{10}$ In our own preliminary experiments, we found that it is easy to get a gain of 300 in a counterpropagating two-beam-coupling configuration. With such a large gain in the two end phase-conjugate mirrors $\left[\mathrm{PCM}_{1}\right.$ and $\mathrm{PCM}_{2}$ of Fig. $\left.2(\mathrm{~b})\right]$ and the large gain in the counterpropagating two-beam coupling in the photorefractive crystal, it should be possible to overcome the losses due to the input and output couplers and to the low diffraction efficiency of the multiple hologram, the spatial filtering, and the Fresnel reflection at each interface. The numbers given above indicate the feasibility of initial experiments involving as many as a few hundred holograms. Another key aspect of the system is the preferential gain exercised by the input beam $E_{i}^{\prime}$ that is nearest the stored message $E_{i}$. An analysis ${ }^{11}$ of the process of phase conjugation by stimulated Brillouin scattering, which is formally analogous to counterpropagation two-beam coupling in photorefractive crystals, shows that the exponential gain constant [condition (1)] for their process varies by as much as a factor of 2 between the cases of spatially correlated or uncorrelated beams. This is expected to introduce a strong preference for the input beam $E_{i}^{\prime}$ that is nearest $E_{i}$ in the winner-take-all environment of a laser.

In conclusion: we have described some basic ideas and phenomena that in combination hold promise for realizing some form of associative memory based on the concept of oscillating message-bearing optical modes. Preferential gain to the desired mode is provided by image-selective two-beam photorefractive coupling between counterpropagating beams.

The authors would like to acknowledge useful discussion on the topic of optical storage in modes of a resonator with Dana Anderson. This research was supported by the U.S. Air Force Office of Scientific Research and the U.S. Army Research Office, Durham, North Carolina. 


\section{References}

1. J. J. Hopfieid, Proc. Nat. Acad. Sci. USA 79, 2554 (1982).

2. M. Cronin-Golomb, B. Fischer, J. O. White, and A. Yariv, IEEE J. Quantum Electron. QE-20, 12 (1984).

3. P. Yeh, Opt. Commun. 45, 323 (1983).

4. Y. H. Ya, Opt. Quantum Electron. 14, 574 (1982).

5. M. Cronin-Golomb, M. B. Fischer, J. Nilsen, and A. Yariv, Appl. Phys. Lett. 41, 220 (1982).

6. A. Yariv, Optical Electronics, 3rd ed. (Holt, Rinehart and Winston, New York, 1985), p. 515.
7. A. Yariv and S. K. Kwong, Opt. Lett. 10, 454 (1985).

8. D.L. Staebler, W. J. Burke, W. Phillips, and J. J. Amodei, Appl. Phys. Lett. 26, 182 (1975).

9. D. Meyerhofer, RCA Rev. 33, 110 (1972).

10. J. Feinberg and R. W. Hellwarth, Opt. Lett. 5, 519 (1980).

11. B. Ya, Zel'dovich, N. F. Pilipetsky, and V. V. Shkunov, Principles of Phase Conjugation, Vol. 42 of Springer Series in Optical Sciences (Springer-Verlag, Berlin, 1985). 